\title{
Recent T2K Neutrino Oscillation Results
}

\author{
H. M. O'Keeffe*, on behalf of the T2K collaboration \\ Physics Department, Lancaster University, UK \\ E-mail: h.okeeffedlancaster.ac.uk
}

T2K is a long baseline neutrino experiment producing a beam of muon neutrinos at the Japan Particle Accelerator Research Centre on the East coast of Japan and measuring their oscillated state $295 \mathrm{~km}$ away at the Super Kamiokande detector. Since 2016, T2K has doubled its data in both neutrino and antineutrino beam modes. Coupled with improvements in analysis techniques this has enabled the experiment to make world leading measurements of the PMNS oscillation parameters $\Delta m_{32}^{2}, \sin ^{2} \theta_{23}$ and the CP violating phase $\delta_{C P}$. In particular the CP conserving values of $\delta_{C P}$ now appear to be disfavoured at the $95 \% \mathrm{CL}$ and there are regions of parameter space excluded at the $99.7 \% \mathrm{CL}$. These results are discussed in this document.

XXIX International Symposium on Lepton Photon Interactions at High Energies - LeptonPhoton2019 August 5-10, 2019

Toronto, Canada

${ }^{*}$ Speaker. 


\section{Introduction}

The phenomenon of neutrino oscillations, first proposed by Pontecorvo in 1957 [1], was confirmed by the observation of atmospheric and solar neutrino oscillations at the Super-Kamiokande [2] and Sudbury Neutrino Observatory [3] experiments. These results, along with those from subsequent experiments, have established a three-flavour mixing framework whereby the three active, light, neutrino flavours are related to three mass states via a unitary mixing matrix referred to as the Pontecorvo-Maki-Nakagawa-Sakata (PMNS) matrix. The PMNS matrix is parameterized by three mixing angles $\theta_{i j}$ (where $i, j=1,2,3$ and $i \neq j$ ) and one CP-violating phase, $\delta_{C P}$. The probability that oscillation will occur is also dependent upon the difference between the squared masses, $\Delta m_{j i}=m_{j}^{2}-m_{i}^{2}(i, j=1,2,3$ and $i \neq j)[4]$.

Both the parameters of the PMNS matrix and the differences between the squared masses can be measured via experiment. This is achieved by measuring the probability of neutrino disappearance, $P\left(v_{x} \rightarrow v_{x}\right)$, or appearance, $P\left(v_{x} \rightarrow v_{y}\right)$, where $x, y=e, \mu, \tau$ and $x \neq y$. All but one of the PMNS matrix parameters have been measured with only the value of $\delta_{C P}$ not known. In addition, there are other measurements still to be made. For example, the order of the mass states i.e. is $m_{1}<m_{2}<m_{3}$ or $m_{3}<m_{1}<m_{2}$ is not known, nor is the octant of $\theta_{23}$. The measurement of the remaining parameters will present a challenge for current, near-future and future oscillation experiments.

\section{The T2K Experiment}

The Tokai to Kamioka (T2K) experiment is a long-baseline neutrino oscillation experiment located in Japan [5]. An intense, beam of predominantly $v_{\mu}$ is produced at the J-PARC facility in Tokai-mura, by colliding $30 \mathrm{GeV}$ protons with a stationary graphite target. The resultant beam of secondary hadrons is comprised of predominantly pions and kaons. A series of three magnetic horns are used to select charged pions which decay in a $96 \mathrm{~m}$ decay volume. Depending on the polarity of the horn current, $\pi^{+}$or $\pi^{-}$can be chosen to produce a $v_{\mu}$ or $\bar{v}_{\mu}$ beam respectively. The neutrino beam is directed at an angle of $2.5^{\circ}$ relative to the axis between the target and the far detector $295 \mathrm{~km}$ away. This "off-axis" technique produces a narrow band beam with a peak energy of approximately $0.6 \mathrm{GeV}$. This corresponds to the energy of the first $v_{\mu} \rightarrow v_{e}$ oscillation maximum for a baseline of $295 \mathrm{~km}$. The number of protons colliding with the graphite target is directly proportional to the flux of (anti)neutrinos. Thus, the number of "protons on target" (POT) is used when referring to the amount of data accumulated by the experiment.

The near detector complex, located approximately $280 \mathrm{~m}$ from the target, consists of an onaxis Interactive Neutrino GRID (INGRID) detector and an off-axis near detector (ND280). The on-axis INGRID detector is used to monitor the direction, position and stability of the beam. The ND280 detector, located $2.5^{\circ}$ off-axis, consists of five sub-detectors; a $\pi^{0}$ detector, two active fine grained detectors, three time projection chambers, an electromagnetic calorimeter and a side muon range detector. These sub-detectors are located inside a magnet, which provides a $0.2 \mathrm{~T}$ field for charge identification and momentum measurement. The ND280 detector is used for measurements of flux and neutrino-nucleus interactions cross-sections. 
The far detector is the Super-Kamiokande water Cherenkov detector, located $295 \mathrm{~km}$ away from the neutrino production point [6]. Super-Kamiokande has a $22.5 \mathrm{kton}$ water fiducial volume surrounded by 11,129 photomultiplier tubes (PMTs). In the water, neutrinos interact via $W$ exchange to produce their corresponding charged lepton partner which, if sufficiently energetic, produces Cherenkov light in the water. Good separation between $v_{e}$ and $v_{\mu}$ candidates is achieved via a particle identification variable, with a probability of misidentifying a $\mu$ as an $e$ of $<1 \%$.

T2K was optimised to perform a precision measurement of $\theta_{23}$ and $\Delta m_{32}^{2}$ via $v_{\mu}$ disappearance and to search for the mixing angle $\theta_{13}$ via $v_{e}$ appearance in the far detector. Having confirmed the appearance of $v_{e}$ in a $v_{\mu}$ beam in 2013 [7], T2K has taken data using both a neutrino and anti-neutrino beam. To July $2018,1.51 \times 10^{21}$ POT in neutrino mode and $1.65 \times 10^{21}$ POT in anti-neutrino mode have collected and a beam power of $500 \mathrm{~kW}$ achieved.

\section{Analysis strategy}

External hadron production data and measurements of the proton beam in-situ are used to predict the (anti)neutrino flux. These are used together with models of interaction cross sections, to generate Monte Carlo simulations of interactions in the near detector, ND280. Selections for different categories of charged-current (anti)neutrino-nucleus interactions are developed using this Monte Carlo data. The selected samples are fitted to the data and the flux, neutrino interaction and detector models are optimised simultaneously to produce the best fit to the measured near detector data. Together with the event rate and kinematic distributions, the best fit flux and neutrino interaction models are propagated to the far detector to provide a prediction of the neutrino energy, neutrino type and event rate distribution at Super-Kamiokande.

The T2K experiment measures the $v_{\mu} \rightarrow v_{\mu}\left(\bar{v}_{\mu} \rightarrow \bar{v}_{\mu}\right)$ disappearance and $v_{\mu} \rightarrow v_{e}\left(\bar{v}_{\mu} \rightarrow \bar{v}_{e}\right)$ appearance probabilities simultaneously to ensure that correlations between the oscillation parameters are accounted for correctly. Three different analyses have been developed to extract the oscillation parameters using either Bayesian or frequentist frameworks. In all cases, the analyses compare the rate and distribution of events in binned kinematic distributions such as reconstructed neutrino energy, reconstructed lepton momentum and reconstructed angle of the outgoing lepton, with predictions tuned to near detector data. The size and correlations of the associated systematic uncertainties are characterized by a covariance matrix. The fit to the near detector data is used to constrain flux and cross-section uncertainties that are common to both the near and far detectors.

\section{Recent results from $T 2 K$}

The T2K experiment has observed the disappearance of $v_{\mu}$ in the neutrino beam mode and $\bar{v}_{\mu}$ in the antineutrino beam mode. The results discussed below, have been derived from a data set of $1.5 \times 10^{21}$ POT in neutrino mode and $1.3 \times 10^{21}$ POT in antineutrino mode. A joint maximum likelihood fit to the far detector data samples is used to extract the oscillation parameters $\sin ^{2} \theta_{23},\left|\Delta m_{32}^{2}\right|, \sin ^{2} \theta_{13}$ and $\delta_{C P}$. Three independent analysis methods are used. Two use a hybrid Bayesian-frequentist approach and the third uses a Markov-Chain Monte-Carlo method. Results from all three analyses are consistent and it is the results from the hybrid Bayesian-frequentist approach using the reconstructed neutrino energy spectrum that are presented here. 
The confidence intervals for the $\left|\Delta m_{32}^{2}\right|-\sin ^{2} \theta_{23}$ plane are shown in Figure 1 for both the normal (black) and inverted (red) mass ordering. A reactor neutrino constraint on $\sin ^{2} \theta_{23}$ has been applied. In the maximum likelihood fit to the far detector event samples, flat priors are chosen for $\sin ^{2} \theta_{23},\left|\Delta m_{32}^{2}\right|$ and $\delta_{C P}$. In the fits where an external, reactor neutrino, measurement is used as a constraint, a Gaussian prior of $\sin ^{2} \theta_{13}$ is used. The best fit value for normal ordering is $\sin ^{2} \theta_{23}=0.536_{-0.046}^{0.031}, \Delta m^{2}=2.343_{-0.072}^{+0.073} \times 10^{-3} \mathrm{eV}^{2}$ and for inverted ordering it is $\sin ^{2} \theta_{23}=0.536_{-0.041}^{0.031}, \Delta m^{2}=2.410_{-0.071}^{+0.070} \times 10^{-3} \mathrm{eV}^{2}$. The data is fully compatible with maximal mixing.

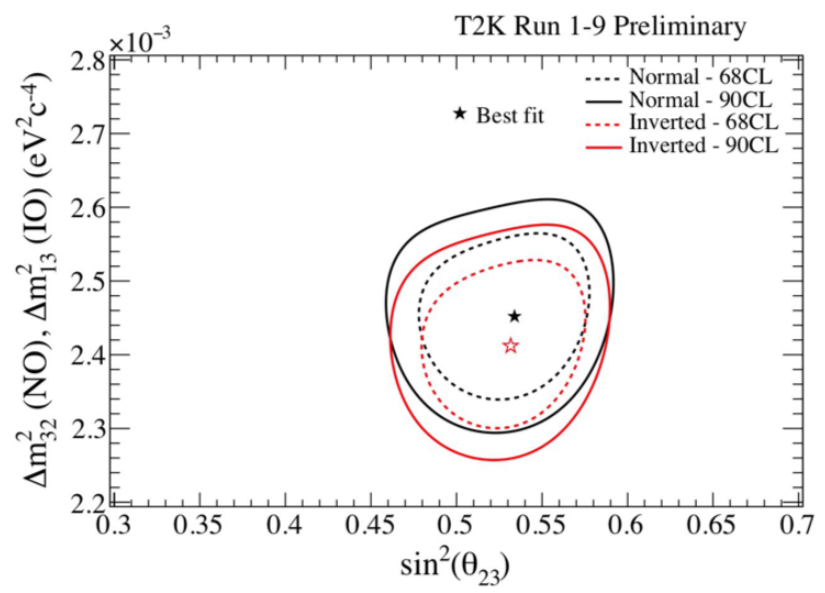

Figure 1: Confidence intervals for the $\sin ^{2} \theta_{23}-\Delta m^{2}$ plane. The reactor neutrino measurement has been used as a prior on $\sin ^{2} \theta_{13}$. The black lines are the intervals for the normal mass ordering hypothesis and those in red are for the inverted mass ordering hypothesis.

Whilst the appearance of $v_{e}$ in a $v_{\mu}$ beam has been well-established by the T2K experiment [7], the results for the search for $\bar{v}_{e}$ appearance in an antineutrino beam are not yet statistically significant. More data are required for a definitive observation of $\bar{v}_{e}$ appearance.

Figure 2 shows the confidence intervals in the $\sin ^{2} \theta_{13}-\delta_{C P}$ plane for the normal and inverted mass ordering, obtained using a reactor neutrino prior on $\sin ^{2} \theta_{13}$. The inclusion of the reactor neutrino prior decreases the size of the confidence intervals and improves sensitivity to $\delta_{C P}$. The Feldman-Cousins method was used to obtain the confidence intervals for $\delta_{C P}$ in Figure 3. The best fit value for normal ordering is $\delta_{C P}=-1.82$ and for inverted ordering it is $\delta_{C P}=-1.38$. The $C P$ conserving values of 0 and $\pi$ are disfavoured at $2 \sigma$ and the the $\delta_{C P} 2 \sigma$ confidence intervals are $(-2.91,-0.64)$ and $(-1.57,-1.16)$ for normal and inverted ordering respectively.

\section{Conclusions}

The T2K experiment measures the oscillation parameters $\theta_{13}, \theta_{23}$ and $\left|\Delta m_{32}^{2}\right|$ and studies $\delta_{C P}$ via a simultaneous analysis of $v_{\mu} \rightarrow v_{\mu}\left(\bar{v}_{\mu} \rightarrow \bar{v}_{\mu}\right)$ disappearance and $v_{\mu} \rightarrow v_{e}\left(\bar{v}_{\mu} \rightarrow \bar{v}_{e}\right)$ appearance probabilities. The current data set comprises $3.1 \times 10^{21}$ POT, split almost equally between neutrino and antineutrino running. 


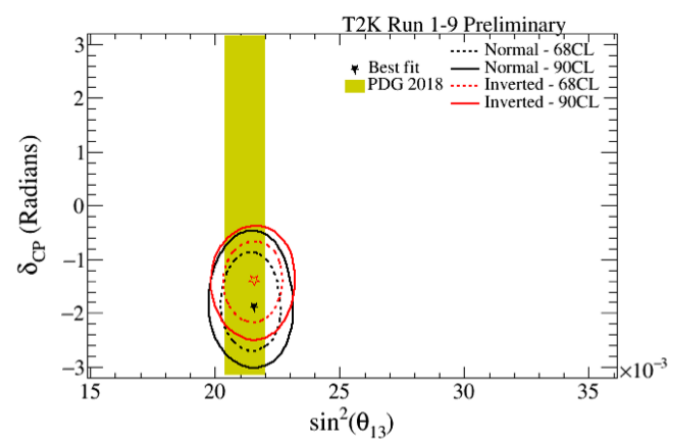

Figure 2: Confidence intervals for the parameters $\sin ^{2} \theta_{13}$ and $\delta_{C P}$. The black lines are the intervals for the normal mass ordering hypothesis and those in red are for the inverted mass ordering hypothesis. The $68 \%$ confidence region from reactor experiments is shown in yellow.

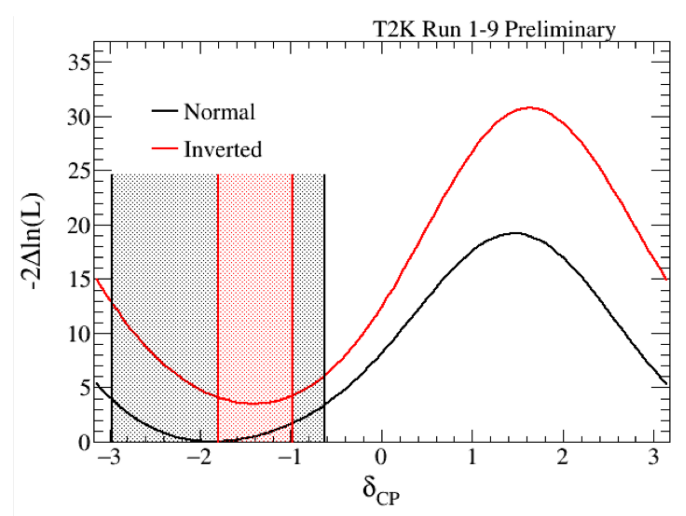

Figure 3: Confidence intervals for $\delta_{C P}$, obtained from the T2K data with a reactor neutrino constraint applied to $\sin ^{2} \theta_{13}$. The vertical lines depict the allowed $2 \sigma$ confidence intervals for the normal (black) and inverted (red) mass orderings. The $C P$ conserving values of 0 and $\pi$ are disfavoured at $2 \sigma$.

Using this data set, the T2K experiment disfavours CP conservation at $2 \sigma$. The best fit results for the parameter $\sin ^{2} \theta_{23}$ are $\sin ^{2} \theta_{23}=0.536_{-0.046}^{0.031}$ for normal mass ordering and $\sin ^{2} \theta_{23}=$ $0.536_{-0.041}^{0.031}$ for inverted ordering. Both results are compatible with maximal mixing. Appearance of $\bar{v}_{e}$ in the antineutrino beam has not yet been confirmed due to statistical limitations. The T2K only measurement of $\sin ^{2} \theta_{13}=0.0268_{-0.0043}^{+0.0055}(N O), 0.0300_{-0.0050}^{+0.0059}(I O)$ is compatible with the reactor only value of $\sin ^{2} 2 \theta_{13}=0.0856 \pm 0.0029$ [8]. By utilizing the reactor only measurement as a constraint in the T2K analysis, a search for $\delta_{C P}$ was made. For both the normal and inverting ordering, the $\mathrm{CP}$ conserving values of 0 and $\pi$ fall outside of the $2 \sigma$ confidence intervals.

The T2K experiment will continue to operate until 2026 and collect $20.0 \times 10^{21}$ POT. Upgrades to the near detector are in progress and together with analysis improvements and increased statistics, $\mathrm{T} 2 \mathrm{~K}$ expects to exclude $\mathrm{CP}$ conserving values by $3 \sigma$ by the end of the experiment. 


\section{References}

[1] B. Pontecorvo, Sov. Physc JETP 6, 429 (1957).

[2] Super-Kamiokande Collaboration, Phys. Rev. Lett. 81 (1998).

[3] SNO Collaboration, Phys. Rev. Lett. 81 (2002).

[4] Z. Maki, M. Nakagawa, S. Sakata, Prog. Theor. Phys. 28 (1962).

[5] T2K Collaboration, Nucl. Instrum. Meth. A 659, 106 (2011).

[6] Y. Fukuda, T. Hayakawa, E. Ichihara, M. Ishitsuka, Y. Itow, et al., Nucl. Instrum. Meth. A 501 (2003).

[7] T2K Collaboration, Phys. Rev. Lett., 112, 061802 (2014).

[8] The Daya Bay Collaboration, Phys. Rev. Lett., 121, 241805 (2018). 